\title{
Desenvolvimento do Pensamento Computacional com o Uso de Internet das Coisas
}

\author{
Carolina Moreira $^{1}$, Eleandro Maschio $^{2}$, Roberto Pereira ${ }^{1}$ \\ ${ }^{1}$ Programa de Pós-Graduação em Informática \\ Universidade Federal do Paraná (UFPR) \\ Curitiba - PR - Brasil. \\ ${ }^{2}$ Coordenação do Curso de Tecnologia em Sistemas para Internet \\ Universidade Tecnológica Federal do Paraná (UTFPR) \\ Guarapuava - PR - Brasil. \\ \{carolmoliveiraa, rpereira.inf\}@gmail.com, eleandromeutfpr.edu.br
}

\begin{abstract}
Technology is embodied into the daily life of society, so it is interesting to learn how to use the resources it offers to increase human intellectual and operational power. Developing the cognitive ability to solve various problems, in many areas of knowledge, is the purpose of Computational Thinking. In this sense, the present research investigates the potential of the Internet of Things as a mediator in the teaching-learning process of Computational Thinking.
\end{abstract}

Resumo. A tecnologia está incorporada ao cotidiano da sociedade, portanto é interessante aprender a utilizar os recursos que ela oferece para aumentar o poder intelectual e operacional humano. Desenvolver a capacidade cognitiva para resolução de diversos problemas, em inúmeras áreas de conhecimento, é a finalidade do Pensamento Computacional. Nesse sentido, a presente pesquisa investiga o potencial da Internet das Coisas como mediadora no processo de ensino-aprendizagem do Pensamento Computacional.

\section{Introdução}

As salas de aula, ao que se observa, permanecem as mesmas de muitos anos atrás, com o professor posicionado em frente à turma, utilizando quadro e giz para transmitir o conhecimento aos alunos [Blikstein 2008]. Em contraste, diversas tecnologias são inerentes ao cotidiano da sociedade moderna, o que pode impactar negativamente na tradicional relação professor-aluno, mas que também abre espaço para novas possibilidades de ensino e aprendizagem.

Segundo [Richter 2000, Piaget 2002], o conhecimento se desenvolve por meio da descoberta. Os autores também afirmam que os alunos precisam ser desafiados durante o processo de ensino-aprendizagem. Logo, é necessário despertar a curiosidade para que se promova a aprendizagem e, desse modo, a informação seja assimilada e o conhecimento, por sua vez, construído. Depois, esse conhecimento pode ser utilizado na resolução de problemas e de desafios reais.

Portanto, existe a necessidade da Educação adaptar-se aos avanços da sociedade e tornar-se mais atraente para os alunos. São recorrentes as pesquisas que enfatizam a importância de trazer as tecnologias atuais para a realidade escolar 
VII Congresso Brasileiro de Informática na Educação (CBIE 2018)

Anais dos Workshops do VII Congresso Brasileiro de Informática na Educação (WCBIE 2018)

[França and Tesdeco 2015, Barcelos and Silveira 2012, Zanetti and Oliveira 2015]. Além disso, introduzir conceitos da Computação no cotidiano escolar tem sido apontado como importante, principalmente para evolução de um novo raciocínio, que é o computacional.

Um conceito eminente, em resposta à problematização descrita, é o Pensamento Computacional, que percebe o uso da Computação como instrumento para favorecer a compreensão do mundo, refletindo naturalmente na produtividade e na criatividade humana. Trata-se de um conhecimento transversal a inúmeras áreas e que se relaciona à Computação enquanto ciência, não ao desenvolvimento de software propriamente dito. Assim, esse conhecimento torna o aprendiz preparado para pensar computacionalmente, conseguindo identificar as tarefas que podem ser realizadas de forma mais rápida e eficiente com o auxílio de um computador [França and Tesdeco 2015].

Em uma rápida busca, são encontrados diversos aplicativos e ferramentas que podem ser utilizados em sala de aula com intuito de ajudar a desenvolver o Pensamento Computacional. São exemplos: Scratch ${ }^{1}$, Alice ${ }^{2}$, App Inventor ${ }^{3}$, Computer Science Unplugged ${ }^{4}$, entre outros. A chegada desses recursos faz com que novas possibilidades possam ser exploradas em sala de aula, principalmente para despertar o interesse dos alunos pelos assuntos e conceitos ensinados.

O desenvolvimento de novas tecnologias está caminhando para a área de Internet das Coisas, na qual a integração de qualquer dispositivo com a rede de computadores permite simplificar e automatizar inúmeras tarefas. O termo emergiu dos avanços de várias áreas, como sistemas embarcados, comunicação e sensoriamento, e a tecnologia já está sendo utilizada em setores como Saúde e Segurança [Mano et al. 2016]. Nesse sentido, a aplicação de Internet das Coisas na Educação abre um leque de novas oportunidades que podem enriquecer o processo de ensino-aprendizagem em diferentes domínios.

Diante disso, o presente artigo objetiva, por meio de uma proposta, discutir sobre a interseção do processo de ensino-aprendizagem do Pensamento Computacional com o potencial da Internet das Coisas. Depois desta introdução, a Seção 2 concentra o referencial teórico da pesquisa. Em seguida, a Seção 3 apresenta uma proposta para desenvolver o Pensamento Computacional com o uso de Internet das Coisas. Por fim, a Seção 4 expõe as considerações feitas até o momento.

\section{Estado da Arte}

O estado da arte da pesquisa alicerça-se em três temas principais, detalhados nas seções seguintes: Pensamento Computacional (2.1), Internet das Coisas (2.2) e Internet das Coisas na Educação (2.3).

\subsection{Pensamento Computacional}

O termo Pensamento Computacional, do inglês Computacional Thinking, foi disseminado por [Wing 2006], como um processo de resolução de problemas utilizando conceitos fundamentais da Ciência da Computação. O estudo apresentou diversos questionamentos

\footnotetext{
${ }^{1}$ Disponível em: https://scratch.mit.edu/

${ }^{2} \mathrm{http}: / /$ www.aliceproject.org/

${ }^{3}$ http://appinventor.mit.edu/

${ }^{4}$ http://csunplugged.org/
} 
VII Congresso Brasileiro de Informática na Educação (CBIE 2018)

Anais dos Workshops do VII Congresso Brasileiro de Informática na Educação (WCBIE 2018)

sobre o que é a Ciência da Computação e como ela pode contribuir em diferentes áreas do conhecimento.

Existem diversas interpretações acerca do termo, porém, desenvolver o Pensamento Computacional não se relaciona somente à competência cognitiva útil para a escrita de algoritmos ou, ainda, a criação de software. O termo está mais associado à construção de um conjunto (bagagem) de conhecimentos que podem ser transpostos para diferentes áreas e aplicáveis em inúmeras situações. Além de que, a capacidade de pensar computacionalmente e raciocinar de maneira lógica é uma prática que fortalece o aprendizado.

A pesquisa de [Zimmermann et al. 2016] ainda afirma que, ao aplicar os conceitos da Ciência da Computação, no momento de explorar uma solução, ajuda-se a desenvolver o pensamento abstrato, algorítmico, lógico e dimensional. Portanto, recursos cognitivos necessários são exercitados a fim de que sejam desenvolvidas soluções para problemas transversais a todas as áreas. Com isso, os sistemas computacionais são vistos como instrumentos para potencializar o poder cognitivo e operacional humano [Blikstein 2008].

O objetivo de desenvolver o Pensamento Computacional está ligado à capacidade de pensar abstratamente em diversos níveis, de maneira independente dos recursos disponíveis. Segundo [Kampff et al. 2016], faz-se necessário identificar as tarefas que precisam ser realizadas de forma mais rápida e eficiente, no sentido de resolver o problema. Essa metodologia envolve um conjunto de procedimentos, dos quais se destacam:

- Abstração: enfoque nas informações importantes, ignorando aquelas desnecessárias para o desenvolvimento da solução;

- Decomposição: simplificação de um problema em problemas menores;

- Desenho algorítmico: escrita da solução do problema, de uma forma eficiente e que procure utilizar a menor quantidade de passos possíveis;

- Reconhecimento de padrões: identificação e agrupamento dos padrões apresentados pelo problema.

Considerando as habilidades mencionadas, percebe-se que desenvolvê-las favorece a autonomia em uma sociedade com inúmeras demandas computacionais. Saber utilizar e tirar vantagem da inteligência humana, associada aos recursos computacionais, aumentará a possibilidade de inserção em um mercado globalizado e de alta competitividade, tendo em vista que a manipulação de softwares, como editores de texto, não é mais suficiente. $\mathrm{O}$ ato de pensar computacionalmente, por consequência, deve abordar fundamentos da Computação enquanto ciência.

Ao desenvolver o Pensamento Computacional, o indivíduo consegue pensar em múltiplos níveis de abstração. Além disso, ele consegue beneficiar-se de recursos computacionais para executar tarefas com maior rapidez e eficiência [Blikstein 2008]. E, em algum tempo, a fim de alcançar esses resultados, o mesmo indivíduo deve ser capaz de programar tais recursos para realizar determinadas tarefas.

\subsection{Internet das Coisas}

O termo Internet das Coisas, do inglês Internet of Things (IoT), se refere a um sistema no qual objetos do mundo físico, utilizando sensores, podem ser conectados à Internet. 
VII Congresso Brasileiro de Informática na Educação (CBIE 2018)

Anais dos Workshops do VII Congresso Brasileiro de Informática na Educação (WCBIE 2018)

Assim, os dispositivos se integram em um ambiente e são capazes de serem identificados, localizados e conectados à Internet, como também de realizar processamento e gerar dados sobre o próprio ambiente e sobre a interação com outros dispositivos [Ashton 2009].

Os objetos presentes no mesmo ambiente conectado possuem a capacidade de trocar informações entre si, influenciar uns aos outros, além de controlar e serem controlados remotamente. Essa interligação permite detectar o contexto em que os objetos se encontram, controlá-los e trocar informações. Ela também possibilita a coleta e o armazenamento de uma grande quantidade de informações geradas por esses objetos [Evans 2011].

O aumento da capacidade de processamento dos dispositivos embarcados, a miniaturização e o barateamento dos sensores têm tornado a IoT cada vez mais aplicável a inúmeros cenários diferentes. A conexão permite expandir as funcionalidades dos objetos comuns, então gerando diversas oportunidades em todas as áreas, tanto no âmbito acadêmico quanto no corporativo e, de maneira transversal, na forma como a sociedade consome informação. São exemplos, entre outros:

1. Ambientes inteligentes;

2. Casas inteligentes;

3. Educação;

4. Segurança;

5. Monitoramento da saúde;

6. Evolução dos carros, transporte e logística; e

7. Controle de focos de incêndio ambiental.

A IoT, em poucas palavras, nada mais é do que a extensão da Internet atual, que proporciona aos objetos do dia-a-dia capacidades extras. Esses objetos, quando interligados, podem ser chamados de objetos inteligentes. Isso ocorre porque os objetos passam a possuir capacidades de comunicação e processamento associadas a sensores, os quais potencializam as suas utilidades originais.

Atualmente, não só computadores convencionais estão conectados à rede, como também uma grande heterogeneidade de equipamentos, tais como TVs, automóveis, eletrodomésticos, telefones, além das possibilidades que aumentam a cada dia. Existem também as tecnologias vestíveis, que são incorporadas em artigos do vestuário, permitindo que as pessoas usem esses objetos e possam realizar outras atividades, como a prática de esportes e os afazeres domésticos. Para que isso fosse possível, os componentes foram sendo miniaturizados, tornando-se imperceptíveis ao uso, de uma maneira que o indivíduo possa sequer cogitar que o objeto tenha certas funcionalidades.

O propósito essencial de interligar dispositivos e coletar dados resume-se em criar consciência da situação. Com isso, permite-se que dispositivos, aplicativos e usuários entendam melhor os ambientes onde se inserem. A compreensão de um cenário habilita os aplicativos a tomarem decisões inteligentes para responderem à dinâmica do contexto [Barnaghi et al. 2012].

Os dispositivos conectados podem gerar uma quantidade absurda de dados. Portanto, a tarefa de processar esses dados e interpretar as informações do mundo real acaba 
VII Congresso Brasileiro de Informática na Educação (CBIE 2018)

Anais dos Workshops do VII Congresso Brasileiro de Informática na Educação (WCBIE 2018)

sendo desafiadora. Porém, depois de analisadas e interpretadas, essas informações podem se transformar em conhecimento, no sentido de proporcionar uma melhor compreensão sobre alguns fenômenos e consequente avanço das soluções oferecidas (ou mesmo científico) [Henson et al. 2009].

\subsection{Internet das Coisas na Educação}

No âmbito educacional, existe uma necessidade por tecnologias que auxiliem no processo de ensino-aprendizagem. A utilização dos sistemas tutores inteligentes, ambientes virtuais de aprendizagem, ferramentas de autoria, smartclasses, ambientes computacionais e smartphones mostram a importância de ferramentas que apoiem esse processo. Mais recentemente, algumas pesquisas buscam investigar o potencial da Internet das Coisas em ambientes educacionais.

Alice das Coisas é um ambiente que foi desenvolvido para analisar a utilização da IoT como mediadora no processo de aprendizagem infantil [Moreira and Baranauskas 2017]. Para isso, foram desenvolvidos objetos e acessórios vestíveis que representavam objetos e fantasias da obra "Alice no País das Maravilhas". Como o principal objetivo era introduzir a tecnologia de uma forma mais natural ao público, buscou-se associar a magia dos contos de fadas à "mágica" dos objetos interconectados.

No mesmo estudo, ainda foram realizadas oficinas com crianças, a fim de que elas explorassem a tecnologia para a construção de narrativas. As crianças podiam interagir com os objetos presentes no cenário, como também utilizar os acessórios vestíveis para se caracterizarem como personagens. Era tanto possível se fantasiar como um personagem da obra, quanto misturar vários acessórios de acordo com a imaginação e compor um personagem novo.

Os objetos presentes no cenário foram confeccionados com etiquetas e leitores RFID. O cenário contava com um computador, uma saída de som e um projetor. Quando dois objetos se comunicavam, o projetor mostrava uma cena do filme "Alice no País das Maravilhas" que envolvesse aqueles objetos. A própria pesquisa afirma que a utilização de um conto de fadas deixou os participantes empolgados e as lembranças do filme guiaram a interação inicial.

Segundo as mesmas autoras, a tecnologia pode ser utilizada para promover a interação social, habilidades de expressão e a formulação do sequenciamento de ideias. Além de que, interagir com coisas que se comunicam proporciona um ambiente colaborativo e rico em possibilidades de criação para a aprendizagem.

A IoT também está sendo utilizada no ensino de Libras. A pesquisa de [Rodrigo Rozendo Bastos 2016] busca oferecer uma experiência agradável que melhore o processo de ensino-aprendizagem da língua brasileira de sinais. Para isso, está sendo desenvolvido um sistema que utiliza objetos vestíveis para captar a movimentação dos dedos e então verificar como está acontecendo o aprendizado. Os movimentos são comparados com outros, armazenados em uma base de dados, para verificar se a representação dos sinais está correta.

Na pesquisa, uma luva de sensoriamento será utilizada para captar os movimentos. A luva pode possuir até 22 sensores de alta precisão que são capazes de perceber as 
VII Congresso Brasileiro de Informática na Educação (CBIE 2018)

Anais dos Workshops do VII Congresso Brasileiro de Informática na Educação (WCBIE 2018)

movimentações para realizar um sinal, o lugar no espaço ou a parte do corpo no qual incide a mão, bem como a respectiva altura e posição. Os dados capturados pela luva serão processados para verificar se o usuário está apresentando um resultado adequado. Dessa forma, a pesquisa usa da tecnologia para promover a inclusão social dos surdos.

\section{Proposta de Pesquisa}

Na literatura, conforme apresentado na Seção 2, as pesquisas sobre Pensamento Computacional são recentes e tornam-se, ainda, mais singulares quando se considera a interseção que utiliza a IoT como recurso. Entretanto, apesar dessa possibilidade quase não ter sido explorada, trata-se de uma abordagem promissora, pois promove novas e interessantes estratégias para o processo de ensino-aprendizagem na área.

Assim, a presente pesquisa investiga o potencial da criação de ambientes educativos (de baixo custo e usando tecnologia livre) com objetos que se comunicam, para o desenvolvimento do Pensamento Computacional, utilizando a IoT como mediadora. A tecnologia será empregada como ferramenta para ampliar a capacidade cognitiva dos alunos em diferentes conteúdos e a interação ajudará a promover o processo de aprendizagem, a tomada de decisões, a interação social e a estruturação de ideias.

Conforme anteriormente descrito, os objetos da IoT são apresentados por alguns autores como "encantados", diante das possibilidades de processamento e de intercomunicação. Por isso, utilizar contos de fadas como temática para elaboração de ambientes educativos pode ser uma metáfora promissora [Moreira and Baranauskas 2017]. Existe potencial nessa temática para ajudar a compreender a correspondência de objetos físicos, no mundo conectado, por meio de uma realidade lúdica que proporcione uma maneira "mágica" de aprender.

A intenção é reavivar o prazer da descoberta que se tem na infância, o qual impulsiona a criança a experienciar o conhecimento e a desafiar-se [Piaget 2002]. Assim, ao utilizar um conto de fadas como temática, para tornar um ambiente educacional mais interessante, procura-se diminuir o impacto dessa interação e deixar os alunos mais seguros para explorarem as possibilidades oferecidas. Por meio da manipulação dos diferentes objetos disponíveis, serão introduzidos, exercitados e reforçados conceitos do Pensamento Computacional, permitindo fundir a experiência do mundo real com o mundo digital.

\subsection{Exemplo}

Harry Potter é uma série de livros escritos pela autora britânica J. K. Rowling. A história narra as aventuras de um jovem chamado Harry James Potter que, aos 11 anos de idade, descobre ser um bruxo quando convidado para estudar na Escola de Magia e Bruxaria de Hogwarts. Um bruxo-feiticeiro é um humano que possui a capacidade de realizar magias, necessitando ou não de uma varinha. Essa capacidade é herdada, porém precisa ser treinada para que o bruxo aprenda a melhor controlá-la.

$\mathrm{Na}$ história existem inúmeras criaturas e objetos mágicos. Os objetos podem ser originalmente mágicos, ou ainda ganhar essa propriedade em um determinado momento. A varinha mágica, por exemplo, é feita de madeira e recheada com uma substância mágica. Assim, a varinha auxilia os bruxos a canalizar seus poderes para realizar feitiços.

Nesse contexto, cenários podem ser idealizados com a temática de Harry Potter, utilizando-se da magia já presente em toda a série. Para um cenário inicial, por exemplo, 
VII Congresso Brasileiro de Informática na Educação (CBIE 2018)

Anais dos Workshops do VII Congresso Brasileiro de Informática na Educação (WCBIE 2018)

os objetos conectados poderiam ser uma varinha mágica e uma pena. O seguinte quadro traz uma possível atividade nesse cenário, inspirada em Computer Science Unplugged:

\section{Atividade: Seguindo Instruções}

Uma das habilidades mais elementares de um bruxo é a levitação ou capacidade de fazer os objetos voarem. Para isso, deve-se realizar o movimento de "girar e sacudir" com a varinha e dizer em voz alta "wingardium leviosa".

Caso o movimento seja realizado de forma errada, um outro feitiço pode ser lançado, fazendo, por exemplo, explodir o objeto que se estava querendo levitar.

Agora é a sua vez! Tente levitar a pena. Utilize o feitiço "wingardium leviosa".

O objetivo da atividade proposta é exercitar o pensamento algorítmico, em que seguir os passos descritos é fundamental para se atingir o resultado esperado. Faz-se analogia a um programa de computador, que opera seguindo um conjunto finito de instruções para cumprir uma determinada tarefa, sendo que um pequeno erro pode causar um comportamento inesperado.

\subsection{Justificativa}

A comunidade de Informática na Educação no Brasil possui, como seu principal evento, o Congresso Brasileiro de Informática da Educação (CBIE), que engloba o Simpósio Brasileiro de Informática na Educação (SBIE) e Workshop de Informática na Escola (WIE). A comunidade ainda mantém a revista Brasileira de Informática na Educação (RBIE), que reúne artigos científicos publicados desde 1997.

A pesquisa de [Posada et al. 2016] mostrou o foco dos trabalhos publicados na RBIE de 1997 a 2014, no SBIE de 2001 a 2014, no WIE em 2003 e entre 2005 e 2014, como também no CBIE de 2012 a 2014. Foi apresentado que a temática dos trabalhos publicados nesses anos permeou as áreas de ensino e aprendizagem, ambientes virtuais, objetos de aprendizagem, mídias digitais, ferramentas de autoria, entre outras. Porém, em nenhum momento, foram registradas pesquisas com o tema de Pensamento Computacional nesses anos.

Ampliando as pesquisas de [Posada et al. 2016], buscou-se, nas edições de 2015, 2016 e 2017 da RBIE, SBIE, WIE e CBIE, publicações com o tema Pensamento Computacional. A Tabela 1 apresenta a quantidade total de artigos publicados por ano, enquanto a Tabela 2 traz a quantidade de artigos sobre o tema citado.

Tabela 1. Quantidade de artigos publicados

\begin{tabular}{c|cccc} 
Ano & RBIE & SBIE & WIE & CBIE \\
\hline $\mathbf{2 0 1 5}$ & 44 & 137 & 67 & 171 \\
$\mathbf{2 0 1 6}$ & 33 & 147 & 103 & 174 \\
$\mathbf{2 0 1 7}$ & 17 & 200 & 134 & 170
\end{tabular}


VII Congresso Brasileiro de Informática na Educação (CBIE 2018)

Anais dos Workshops do VII Congresso Brasileiro de Informática na Educação (WCBIE 2018)

Tabela 2. Quantidade de artigos publicados sobre Pensamento Computacional

\begin{tabular}{c|cccc} 
Ano & RBIE & SBIE & WIE & CBIE \\
\hline $\mathbf{2 0 1 5}$ & 0 & 4 & 6 & 18 \\
$\mathbf{2 0 1 6}$ & 2 & 9 & 22 & 17 \\
$\mathbf{2 0 1 7}$ & 0 & 11 & 23 & 27
\end{tabular}

Analisando-se os números apresentados nas tabelas 1 e 2, tem-se que, ao todo, foram publicados 1.397 artigos nos principais veículos científicos nacionais. Desses, 139 abordavam o tema do Pensamento Computacional, totalizando $10 \%$ das publicações. Portanto, percebe-se que o referido tema é relevante pela quantidade crescente de publicações que o abordam.

Com o crescimento do tema, foi promovido o Workshop de Ensino em Pensamento Computacional, Algoritmos e Programação (WAlgProg). Em 2017, o evento teve sua terceira edição e já publicou 92 artigos, representando 18\% dos artigos do CBIE. Nas três edições, movimentou 44 artigos com enfoque específico no Pensamento Computacional. A maioria desses trabalhos associava o Pensamento Computacional com:

- Conceitos matemáticos;

- Scratch;

- Jogos; e

- Robótica.

De todo o conjunto de publicações entre os anos de 2015 e 2017 (Tabela 1), verificou-se ainda que poucos artigos uniam a Internet das Coisas e a Educação. Constatou-se, também, a carência de pesquisas que reunissem o desenvolvimento do Pensamento Computacional e o conceito de Internet das Coisas. Por fim, justifica-se que, embora as pesquisas em Pensamento Computacional tenham crescido na última década, não se trata de um conceito efetivamente difundido e explorado. Observam-se, então, indícios de que existe espaço significativo para pesquisas que busquem desenvolver o Pensamento Computacional utilizando a IoT.

Além de tudo isso, a capacidade de pensar computacionalmente contribuirá para o acesso participativo e universal do cidadão brasileiro ao conhecimento, citado como o $4^{\circ}$ dos Grandes Desafios da Computação no Brasil [SBC 2006]. A computação ubíqua pode se tornar um fator de exclusão e divisão de classes, possuindo potencial para criar pessoas incapazes de viver autonomamente em um ambiente mediado por TICs, aprofundando a desigualdade. Portanto, desenvolver o Pensamento Computacional é uma forma de favorecer a autonomia e o acesso ao conhecimento.

\section{Considerações Finais}

A Computação constitui uma área de conhecimento que permeia quase todas as atividades humanas na sociedade, de forma que não se pode imaginar um futuro sem tecnologia. Nesse sentido, passa a ser inconcebível a imagem de um cidadão ignorante em Computação, uma vez que, em qualquer ofício, haverá o uso de tecnologias. Inúmeros problemas, em diferentes áreas, podem ser (melhor) resolvidos ao se pensar computacionalmente. 
VII Congresso Brasileiro de Informática na Educação (CBIE 2018)

Anais dos Workshops do VII Congresso Brasileiro de Informática na Educação (WCBIE 2018)

Estimular o Pensamento Computacional ajuda a desenvolver competências e habilidades fundamentais para o domínio da tecnologia. Os dispositivos computacionais estão integrando o cotidiano da sociedade e, mais que isso, vêm sendo incorporados em diversos objetos. Assim, a sociedade está testemunhando o início de uma nova era, em que aprender a utilizar os recursos disponibilizados pela tecnologia reflete naturalmente no poder intelectual e operacional humano [Blikstein 2008].

Por tudo isso, o presente artigo, por meio de uma proposta, enfocou o desenvolvimento do Pensamento Computacional e expôs argumentos para investigar a utilização da Internet das Coisas como mediadora nesse processo. Acredita-se que essa discussão inicial com a comunidade do WAlgProg seja promissora e possa trazer contribuições relevantes para a área.

\section{Agradecimentos}

O presente trabalho foi realizado com apoio da Coordenação de Aperfeiçoamento de Pessoal de Nível Superior - Brasil (CAPES) - Código de Financiamento 001.

Os autores agradecem a Universidade Tecnológica Federal do Paraná, Câmpus Guarapuava, pelo suporte financeiro, por meio do Edital 01/2018 - DIRPPG-GP, para apresentação do artigo.

\section{Referências}

Ashton, K. (2009). That 'Internet of Things' Thing. RFID Journal.

Barcelos, T. S. and Silveira, I. F. (2012). Pensamento Computacional e Educação Matemática: Relações para o Ensino de Computação na Educação Básica. In $X X$ Workshop sobre Educação em Computração (WEI).

Barnaghi, P., Wang, W., Henson, C., and Taylor, K. (2012). Semantics for the Internet of Things: Early progress and back to the future. In Proceedings of the International Journal on Semantic Web and Information Systems (IJSWIS).

Blikstein, P. (2008). O Pensamento Computacional e a Reinvenção do Computador na Educação. http://www.blikstein.com/paulo/documents/online/ol_pensamento_computacional.html.

Evans, D. (2011). The Internet of Things: How the Next Evolution of the Internet is Changing Everything. Cisco White Paper.

França, R. and Tesdeco, P. (2015). Desafios e oportunidades ao ensino do Pensamento Computacional na Educação Básica no Brasil. In Anais dos Workshops do IV Congresso Brasileiro de Informática na Educação (CBIE 2015), pages 1464-1473.

Henson, C. A., Pschorr, J. K., Sheth, A. P., and Thirunarayan, K. (2009). SemSOS: Semantic Sensor Observation Service. In Proceedings of the 2009 International Symposium on Collaborative Technologies and Systems, CTS '09, pages 44-53, Washington, DC, USA. IEEE Computer Society.

Kampff, A. J. C., Lopes, T. R. C., da Rosa Alves, I. M., de Souza, V. C., Marson, F. P., and Rigo, S. J. (2016). Pensamento Computacional no Ensino Superior: Relato de uma oficina com professores da Universidade do Vale do Rio dos Sinos. In Anais 
VII Congresso Brasileiro de Informática na Educação (CBIE 2018)

Anais dos Workshops do VII Congresso Brasileiro de Informática na Educação (WCBIE 2018)

dos Workshops do V Congresso Brasileiro de Informática na Educação (CBIE 2016), pages 1316-1323.

Mano, L., Funes, M., Volpato, T., and Neto, J. (2016). Explorando tecnologias de IoT no contexto de Health Smart Home: uma abordagem para detecção de quedas em pessoas idosas. Journal on Advances in Theoretical and Applied Informatics, 2(1):46-57.

Moreira, E. A. and Baranauskas, M. C. C. (2017). Alice das Coisas: entendendo a comunicação entre objetos na construção de ambientes de aprendizagem. Anais do XXVIII Simpósio Brasileiro de Informática na Educação (SBIE 2017), pages 10171026.

Piaget, J. (2002). Epistemologia Genética. WMF Martins Fontes, São Paulo.

Posada, J. E. G., Buchdid, S. B., and Baranauskas, M. C. C. (2016). A Informática na Educação: O que Revelam os Trabalhos Publicados no Brasil. Revista Brasileira de Informática na Educação, 24(1):141-155.

Richter, M. G. (2000). Ensino de Português e Interatividade. Editora UFSM, Santa Maria.

Rodrigo Rozendo Bastos, Lucas Lins, R. d. A. S. (2016). Aperfeiçoando o aprendizado da Libras utilizando elementos de Internet das Coisas. Anais dos Workshops do V Congresso Brasileiro de Informática na Educação (CBIE 2016), pages 1364-1373.

SBC (2006). Grandes Desafios da Computação no Brasil. Technical report, Sociedade Brasileira de Computação.

Wing, J. M. (2006). Computational Thinking. Communications of the ACM, 49(3):33-35.

Zanetti, H. and Oliveira, C. (2015). Prática de ensino de Programação de Computadores com Robótica Pedagógica e aplicação de Pensamento Computacional. In Anais dos Workshops do IV Congresso Brasileiro de Informática na Educação (CBIE 2015), pages 1236-1245.

Zimmermann, J. S. O., Watanabe, A., Barcelos, T. S., and Mancini, F. (2016). Proposta de aplicação e avaliação de conceitos do Pensamento Computacional em crianças hospitalizadas. In Anais dos Workshops do V Congresso Brasileiro de Informática na Educação (CBIE 2016). 